\title{
Elephantiasis nostras verrucosa in a patient with lipedema and lipolymphedema
}

\section{Lívia Maria Pereira de Godoy', Paula Pereira de Godoy Capeletto', Ana Carolina Pereira de Godoy², Jose Maria Pereira de Godoy ${ }^{3}$}

\author{
${ }^{1}$ Physician Specialized in General Practice, Researcher Group of the Clínica Godoy, São José do Rio Preto, SP-Brazil \\ ${ }^{2}$ Physician Specialized Intensive Therapy, Research Group of the Clinica Godoy, São Jose do Rio Preto, Brazil, ${ }^{3}$ Professor \\ Adjunt of Cardiology and Cardiovascular Surgery Department of the Medicine School in São José do Rio Preto-FAMERP, \\ Research CNPq (National Council for Research and Development), Brazil
}

Corresponding author: Dr. Lívia Maria Pereira de Godoy, E-mail: godoyjmp@gmail.com

\begin{abstract}
Elephantiasis nostras verrucosa is a group of rare cutaneous changes comprising dermal fibrosis, hyperkeratotic, and verrucous and papillomatous lesions secondary to chronic non-filarial lymphedema. The following study reports a case associated with lipedema and lymphedema, several episodes of erysipelas unfolding over the previous years, and warty lesions appearing in the transitional region between the right ankle and foot. Several episodes of erysipelas in patients with lipolymphedema can evolve into elephantiasis nostras verrucosa (ENV).
\end{abstract}

Key words: Elephantiasis nostras verrucosa; Lipedema; Lymphedema

\section{INTRODUCTION}

Elephantiasis nostras verrucosa (ENV) is a group of rare cutaneous changes comprising dermal fibrosis, hyperkeratotic, and verrucous and papillomatous lesions secondary to chronic non-filarial lymphedema [1]. It is a peculiar clinical stage of lymphedema resulting from infectious processes in the limbs and leading to their fibrosis. It is also a progressive condition that, without intervention, leads to continuing deformity and disability [2]. However, there is no standard treatment for ENV, and surgery may not always be its best treatment since it does not treat its cause, only its cutaneous symptoms. Nevertheless, debridement is a quick and aesthetically adequate form of treatment [3].

Lipedema is a clinical syndrome first described by Allen and Hines in 1940 [1] that is characterized by a bilateral and symmetrical increase in the size of the lower limbs, but not the feet. Its physical features are characterized by a more pronounced deposition of fat tissue in the extremities. Stemmer's sign is negative. Its signs and symptoms may include, among other conditions, cutaneous hypothermia, alterations in the plantar support, and hyperalgesia [4,5]. It is important to understand that the concept of lipolymphedema is a progression of lipedema to lymphedema [6]. The aim of this study is to report on the association of lipedema with elephantiasis, the most advanced clinical stage of lymphedema, and elephantiasis nostra verrucosa.

\section{CASE REPORT}

The patient reported that the swelling had been persistent for around five years without diminishing upon rest and was, therefore, diagnosed as lipolymphedema. The warty lesions on the right ankle and foot (Fig. 1) were diagnosed as elephantiasis nostras verrucosa, the advanced clinical stage of lymphedema. There were no obvious clinical signs of lymphedema of the left foot at the time of the physical examination (Fig. 2), but there was an ulcerated lesion due to infection. Lesions were also observed in the middle third of the right leg, suggestive of grade II lipedema. The case received approval from the Human Research Ethics 
Committee of São José do Rio Preto School of Medicine (\#2.336.797).

\section{DISCUSSION}

This report describes elephantiasis nostras verrucosa in a patient with an evolution of lipedema into lipolymphedema. This type of presentation of lipedema has not been described in the literature. The term 'nostras' was introduced by Castellani in order to distinguish tropical filarial elephantiasis from lymphedematous disorders in the temperate regions. One suggested hypothesis for the etiology and pathogenesis of the disease is lymphangitis and localized lymphatic lesions caused by streptococcal infection [7].

In this study, the patient reported several outbreaks of erysipelas, which had probably contributed to the aggravation of the lipedema. Initially, these outbreaks were localized and led to the development of elephantiasis nostra. Subsequently observed was evolution of lipolymphedema. This finding highlights the consequences of chronic infection in the evolution of this type of disease.

Lipedema is a common condition that involves the distribution of fat tissue and leads to changes in the lymphatic system [8]. The possibility that prior lymph injury or recurrent infection may be its cause should, however, be questioned.

As for treatment, the use of bandages is inadvisable because the friction on the verrucosities may cause injury and infection. The patient reported that this change occurred during one of the treatments. Clinical management does not, therefore, appear to be effective in more advanced cases of the disease, and surgical resection of the lesions to permit the use of bandages seems, currently, to be the best option. In this case, prophylaxis with benzathine penicillin reduced the outbreaks of infection, but, on cessation, the infections restarted.

\section{Consent}

The examination of the patient was conducted according to the principles of the Declaration of Helsinki.

The authors certify that they have obtained all appropriate patient consent forms, in which the patients have given consent for images and other clinical information to be included in the journal. The patients understand that their

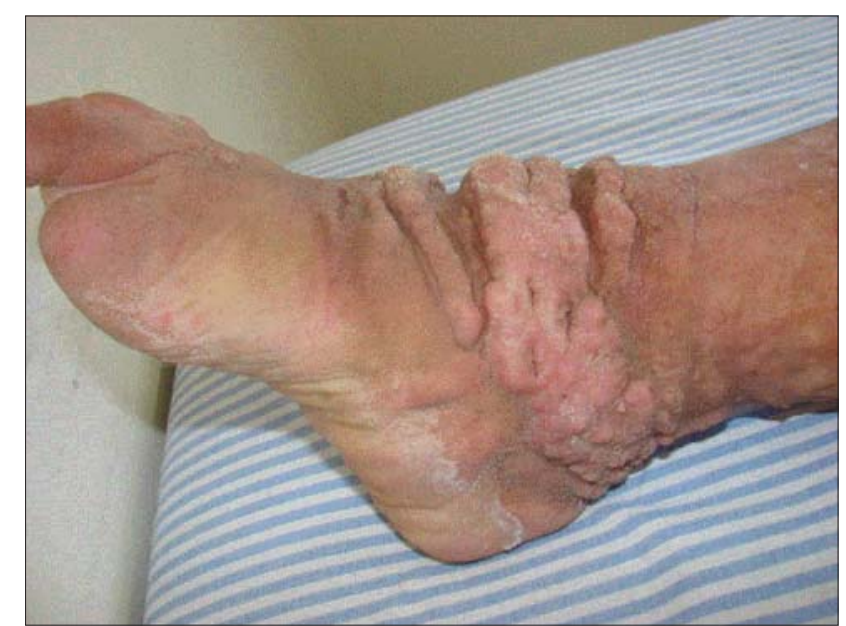

Figure 1: The right ankle and foot with lipolymphedema and elephantiasis nostras verrucosa; advanced fibrosis and nodules on the lower third of the leg, suggestive of grade II lipedema.

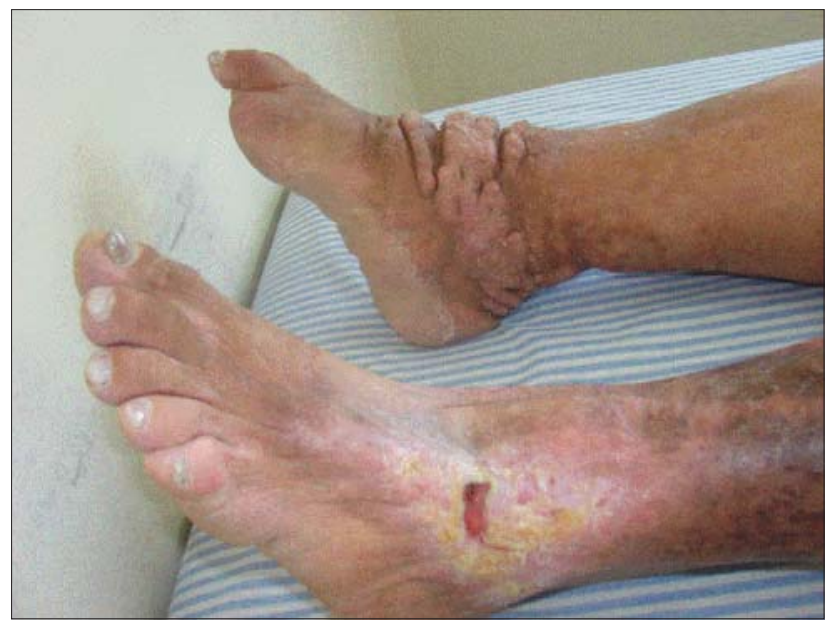

Figure 2: The lower limbs with lipolymphedema, advanced elephantiasis nostras verrucosa on the right leg, and an ulcerated lesion on the left leg.

names and initials will not be published and due effort will be made to conceal their identity, but that anonymity cannot be guaranteed.

\section{REFERENCES}

1. Liaw FY, Huang CF, Wu YC, Wu BY. Elephantiasis nostras verrucosa: swelling with verrucose appearance of lower limbs. Can Fam Physician. 2012;58:e551-3.

2. Borman P. Lymphedema diagnosis, treatment, and follow-up from the view point of physical medicine and rehabilitation specialists. Turk J Phys Med Rehabil. 2018;64:179-97.

3. Kar Keong N, Siing Ngi AT, Muniandy P, Voon Fei W. Elephantiasis nostras verrucosa: a rare complication of lower limb lymphoedema. BMJ Case Rep.2017;2017:bcr2017221492.

4. Allen E V, Hines E A, Lipedema of the legs. A syndrome characterized by fat legs and edema. Proc Staff Meat Mayo Clin. 1940;15:184.

5. Pereira de Godoy JM, Pereira de Godoy HJ, de Sene Souza AA, Budtinger Filho R, de Fatima Guerreiro Godoy M. Lipolymphedema 
www.odermatol.com

associated with idiopathic cyclic edema: a therapeutic approach. Case Rep Vasc Med. 2017;2017:5470909.

6. Pereira De Godoy JM, Augusto Dos Santos R, Vilela Filho RA, Guerreiro Godoy Mde F. Erysipelas and ulcer of the legs in patients with lipolymphedema. Eur J Dermatol. 2011;21:101-2.

7. Shah FY, Bhat YJ, Zeerak S, Wani R, Hassan Shah I. Cutaneous mastocytosis mimicking hypertrophic scars: A case report. Our Dermatol Online. 2020;11:50-2.
8. Amann-Vesti BR, Franzeck UK, Bollinger A. Microlymphatic aneurysms in patients with lipedema. Lymphology. 2001;34:170-5.

Copyright by Lívia Maria Pereira de Godoy, et al. This is an open access article distributed under the terms of the Creative Commons Attribution License, which permits unrestricted use, distribution, and reproduction in any medium, provided the original author and source are credited.

Source of Support: Nil, Conflict of Interest: None declared. 